\title{
ETUDE ZOOLOGIQUE DE LA LARVA MIGRANS
}

\section{Par Claude PETTER}

\section{Introduction}

Depuis quelques années, les auteurs de plusieurs pays ont signalé des syndromes cliniques mystérieux où coexistent une forte éosinophilie, des lésions granulomateuses du foie, des infiltrats pulmonaires.

Certains les assimilaient au syndrome de Weingarten, au syndrome de Frimodt-Moeller, à l'éosinophilie tropicale, mais le diagnostic étiologique restait souvent négatif.

Quelques auteurs réussirent à prouver la présence de migrations larvaires dans les tissus, chez le nourrisson, de certains Ascaridiens du Chien et du Chat.

On peut se demander si des cas d'infestation analogue ne sont pas plus fréquents qu'on ne le pense, et s'ils ne se produisent pas même chez l'adulte.

On pourrait certainement élargir le nombre des Ascaridiens pouvant accidentellement infester l'homme.

Nous nous proposons ici de rappeler les grandes lignes de la Biologie des Ascaridiens pouvant être pathogènes pour l'homme. Dans une seconde partie, nous essaierons d'établir une liste des espèces d'Ascaridiens de nos pays qui, par l'écologie de leur hôte définitif, peuvent être transmis facilement à l'homme et occasionner des troubles tissulaires.

Enfin, dans une troisième partie, nous présenterons un certain nombre de cas cliniques relatés dans la littérature médicale. 


\section{Biologie des Ascaridiens}

Pour étudier ce chapitre, nous nous sommes appuyé sur les travaux de A.-G. Chabaud de 1955, avec les corrections qu'il nous a suggérées, car des notions nouvelles l'ont conduit à remanier profondément le plan qui avait été adopté à cette époque.

Nous savons que le développement post-embryonnaire de tous les Nématodes parasites phasmidiens comporte cinq stades successifs, la larve muant quatre fois. La larve infestante pour l'hôte définitif est au début du $3^{\circ}$ stade.

Plus précisément chez les Ascaridiens, la première mue s'effectue le plus souvent dans l'œuf; la larve infestante est presque toujours au début du $3^{-}$stade, mais parfois le cycle se trouve raccourci par suppression de l'hôte intermédiaire, et c'est le $2^{\circ}$ stade qui est infestant pour l'hôte définitif.

Chez la plupart des Ascaridiens, nous avons affaire à des parasites hétéroxènes, c'est-à-dire qui nécessitent le passage de stades larvaires dans un hòte intermédiaire ; ainsi, dans le cas des Ascaridiens pathogènes pour l'homme, qui nous intéressent surtout ici, cet hôte intermédiaire sera le plus souvent infesté par ingestion du $2^{\circ}$ stade larvaire qui effectuera des migrations à travers les tissus de l'hôte intermédiaire, finissant par s'enkyster au $3^{\circ}$ stade dans les tissus (cas de l'Ascaris devosi). L'hôte définitif s'infestera en ingérant l'hôte intermédiaire.

Il ne faut pas confondre cet hòte intermédiaire infesté par le $2^{c}$ stade larvaire avec l'hôte d'attente qui pourrait se substifuer à l'hôte définitif. L'hòte d'attente ou de réencapsulement ne s'infestera qu'avec le $3^{\text {e }}$ stade larvaire et les larves s'enkysteront dans ses tissus en n'y subissant aucun développement. Il pourra y avoir successivement plusieurs hôtes d'attente.

Les larves du $2^{\circ}$ stade ont donc un ròle actif dans les tissus de l'hôte intermédiaire, à travers lesquels elles migrent, et c'est au cours de ces migrations qu'elles occasionneront chez l'hôte intermédiaire un certain nombre de troubles, parfois graves, dus au métabolisme actif présenté par les larves au cours de leur croissance du $2^{\circ}$ au $3^{\circ}$ stade.

Pour comprendre la biologie des Ascaridiens, il est nécessaire de connaitre une notion très importante : la notion de séclusion.

On observe chez les Nématodes une évolution tendant à une adaptation des larves à un milieu de plus en plus éloigné du milieu extérieur, e'est-à-dire à une séclusion de plus en plus forte des 
stades larvaires; ainsi, nous verrons se dessiner des cycles qui se compliquent progressivement en partant des larves libres, puis des larves ayant la première mue à l'intérieur de l'œuf; nous voyons ensuite des larves se développant chez un hôte intermédiaire à séclusion faible : Mollusque, Oligochète, etc..., des larves se développant dans un hòte intermédiaire à séclusion forte : Arthropodes, puis des Vertébrés interviennent.

Nous allons vérifier cela en passant en revue les différents types offerts par les Ascaridiens, en allant des plus simples aux plus évolués.

\section{Il n'y a pas d'hôte intermédiaire ou, s'il en existe un, il a une séclusion faible.}

Au bas de l'échelle, nous placerons les Cosmocercoidea qui ne comportent pas de mue à l'intérieur de l'œuf.

Chez ces Nématodes, les trois premiers stades larvaires sont soit libres dans le milieu extérieur, soit chez un hôte intermédiaire à séclusion faible, par exemple un Mollusque, un Oligochète, chez lequel ils évoluent.

\section{Il y a un hôte intermédiaire à séclusion faible.}

Plus élevé dans l'évolution, le Porrocæcum crassum, étudié par Mosgovoi (1952), montre une première mue dans l'œuf. Là encore, l'hôte intermédiaire utilisé a une séclusion faible : Ver de terre. Les œufs de Porrocæcum crassum, cultivés à $22^{\circ} \mathrm{C}$., donnent des larves qui muent dans l'œuf après quelques jours. L'infestation directe était impossible.

Mosgovoi découvrit que l'œuf était ingéré par le Ver de terre ; après éclosion dans l'intestin de ce dernier, les larves migrent dans les vaisseaux où elles muent et grandissent en devenant infestantes.

Quand le Ver de terre est dévoré par le Canard, les larves sont libérées et entrent dans la sous-muqueuse du gésier qu'elles quittent après sept jours. Elles passent ensuite dans le grêle et arrivent à maturité en trois semaines.

Nous n'avons donc ici qu'un seul hòte intermédiaire.

Récemment, G. Osche a montré le cycle analogue de Porrocæcum ensicaudatum, parasite du Merle et de l'Etourneau.

L'œuf embryonné est ingéré par le Ver de terre, la larve migre dans ses vaisseaux sanguins, à l'intérieur desquels elle effectue sa croissance, et infeste à son $3^{\circ}$ stade l'Oiseau qui ingère le Ver de terre. 


\section{Il y a un hôte intermédiaire, à séclusion faible, et un second hôte intermédiaire vertébré.}

Gas de Porrocæcum talpæ.

Dans le cas de Porrocæcum talpæ, étudié par G. Osche, on peut voir l'association possible d'un invertébré et d'un vertébré jouant le rôle d'hôtes intermédiaires. On trouve le parasite adulte dans le tube digestif des Rapaces diurnes et nocturnes. En disséquant des Musaraignes de diverses espèces, G. Osche a trouvé des larves encapsulées dans le mésentère et, fait qui nous paraît très important, il a pu trouver une larve encapsulée, beaucoup plus petite que les autres (environ la moitié), ce qui permet de penser que les larves effectueraient une migration en croissant dans les tissus de l'Insectivore avant de s'encapsuler.

Le fait que les Vers de terre soient souvent hôtes intermédiaires chez d'autres espèces de Porrocæcum permet de penser qu'ils jouent un rôle comme premier hòte intermédiaire ; d'autre part, la Musaraigne, se nourrissant de Lombrics, s’infesterait très facilement et pourrait jouer le rôle du deuxième hôte intermédiaire.

Toutefois, il est possible que le vertébré ici aussi soit seul nécessaire pour boucler le cycle.

\section{Il y a un hôte intermédiaire, à séclusion forte, et un second hôte intermédiaire vertébré.}

Gas de Raphidascaris canadensis.

Chez ce parasite du Brochet, étudié par Thomas en 1937, la première mue se ferait dans l'œuf; la larve du $2^{\text {e }}$ stade est infestante pour l'hôte intermédiaire. Si l'œuf est ingéré par une nymphe d'Odonate, la larve y éclôt et le $2^{\circ}$ stade y entreprend sa croissance.

Il semble, d'après Thomas, que la larve ne puisse arriver au $3^{\circ}$ stade que si la Libellule est dévorée par un petit Poisson.

Thomas a montré que l'invertébré n'était pas nécessaire pour fermer le cycle, toute l'évolution larvaire pouvant se faire chez le vertébré hôte intermédiaire. De même, l'évolution du parasite de Cormoran Contracæcum spiculigerum (Rud.) a été réussie par cet auteur avec seulement des petits vertébrés (Têtards, Poissons).

Il est possible que l'invertébré soit obligatoire dans certains cycles ; ainsi, Teixeira de Freitas et Lent (1946) signalent l'infestation naturelle de Diaptomus par le $2^{\circ}$ stade de Goezia spinulosa (Diesing). (Dans ce cas, l'hôte intermédiaire a encore une séclusion assez faible).

Ann. de Parasitologie, T. XXXV, ${ }^{\circ} 1-2,-1960$. 


\section{Il n'y a qu'un seul hôte intermédiaire vertébré.}

Chez certaines espèces terrestres, l'hôte intermédiaire vertébré est obligatoire et le cycle direct est impossible : c'est le cas étudié par Sprent (1952) chez l'Ascaris devosi, qui évolue dans le tube digestif du Furet.

L'œuf se segmente dans le milieu extérieur ; dès le $6^{\circ}$ jour, il contient une larve mobile ; la première mue se fait vers le $12^{\circ}$ jour et le $2^{\prime \prime}$ stade devient infestant pour l'hôte intermédiaire.

A ce stade, si l'œuf est ingéré par un petit Rongeur, il éclòt dans l'intestin; la larve traverse la paroi du tube et migre dans les muscles et les viscères, où elle mue en augmentant de taille, et atteint le $3^{\circ}$ stade infestant encapsulé.

Le Furet s'infeste en dévorant le Rongeur et les larves muent, cette fois, sans aucune migration dans l'intestin du Furet hôte définitif.

Il en est de même dans le cas de l'Ascaris columnaris, parasite de Procyonidés et de Viverridés, où l'hôte définitif s'infeste en dévorant des Rongeurs parasités.

Chez d'autres espèces, bien que jamais constaté, le cycle indirect paraît certain :

Les larves d'Ophidascaris filaria (Dujardin) (Sprent, 1953), parasite de Serpents, grandissent rapidement dans les tissus de la Souris où elles finissent par s'enkyster dans les muscles des pattes antérieures, mais atteignent leur taille maximum dans le foie.

A.-G. Chabaud, ayant expérimenté sur Hexametra, parasite de la Vipère de France, est arrivé à des conclusions analogues ; probablement, les larves développées chez la Vipère, infestée expérimentalement par des œufs introduits dans l'estomac par tubage, resteraient bloquées au $3^{e}$ stade et, là encore, le cycle direct serait impossible.

Chez d'autres espèces, le Vertébré hôte intermédiaire ne sera plus obligatoire, mais facultatif, au cours du cycle évolutif ; on en connaît de nombreux cas :

L'Ascaris du Chien, Toxocara canis, évolue pendant ses stades larvaires chez la Souris, effectuant des migrations tissulaires. Le Chien pourra s'infester en dévorant la Souris (Sprent, 1953), mais cette espèce peut aussi avoir un cycle direct; dans ce cas, l'hôte intermédiaire est éliminé.

De même pour l'Ascaris du Lion, Ascaris leonina, et l'Ascaris transfuga de l'Ours noir, où nous voyons un cycle comparable chez 
la Souris ; mais, ici, la maturation du $2^{\circ}$ stade se fera dans l'épaisseur de la paroi intestinale.

Le Chat peut s'infester en dévorant la Souris parasitée (Sprent, 1953).

6. Les migrations, chez l'hôte définitif, remplacent celles qui se déroulaient chez l'hôte intermédiaire.

Gas des Ascaridiens monoxènes: Ascaris lumbricoides par exemple.

Chez ces Ascaridiens, nous assistons à la disparition totale du vertébré hôte intermédiaire ; il faut voir là des parasites plus évolués dérivant de cycles hétéroxènes avec un retour à la monoxénie. C'est le cas de l'Ascaris lumbricoides, parasite de l'Homme, de Neoascaris vitulorum, évoluant chez le Veau, et de Parascaris equorum (Goeze) du Cheval.

L'hôte définitif, l'Homme par exemple dans le cas de l'Ascaris lumbricoides, s'infeste en ingérant les larves au début de leur $2^{\circ}$ stade et joue le rôle d'hôte intermédiaire.

La migration qui conduit les larves aux poumons de l'homme correspond à celle qui s'effectuait chez la Souris dans le cas de l'Ascaris devosi par exemple.

Le cycle évolutif de l'Ascaris de l'homme est relativement simple : l'œuf embryonné étant évacué dans les selles, l'embryon se développe en 30 à 40 jours lorsque la température est suffisamment élevée.

La larve reste dans l'œuf ; c'est à partir de l'œuf embryonné que l'homme contracte l'Ascaridiose.

Il y a une mue dans l'œuf et l'embryon infestant est au $2^{e}$ stade larvaire. L'œuf ingéré par l'homme passe dans le tube digestif et, sous l'influence du suc gastrique, la coque se laisse percer et la larve en sort.

Celle-ci, incapable de se développer sur place, traverse les parois digestives et tombe dans les vaisseaux sanguins qui la portent au cœur ; celui-ci la chasse dans les poumons où elle séjourne assez longtemps, augmente de taille et, au bout d'une semaine environ, remonte dans la trachée.

Elle est ensuite avalée avec les mucosités bronchiques et revient dans l'intestin où elle se fixe et devient adulte.

On comprend facilement que ces Ascaridiens évolués soient peu dangereux pour l'homme. En effet, bien que ces parasites soient très facilement en contact avec l'homme, puisqu'ils infestent des 
animaux domestiques, il est rare qu'ils puissent être pathogènes pour l'homme; les infestations non pathogènes chez l'homme sont certainement plus courantes qu'on ne l'imagine, mais les larves n'évoluant pas dans les tissus ne donneront que peu de signes cliniques, ces parasites étant très spécifiques vis-à-vis du choix de l'hôte définitif, et les jeunes larves de l'Ascaris du Veau, de celui du Cheval ou de l'Ascaris suum du Porc, ingérées accidentellement par l'homme, ne migreront pas dans les tissus humains, ou, si elles y migrent, leur croissance ne s'effectue pas et leur métabolisme étant pratiquement nul, l’organisme humain ne sera pas sensibilisé.

\section{Il n'y a pas d'hôte intermédiaire, la migration se simplifie ou disparaît.}

Comme chez Heterakis, l'œuf mûr contient un jeune $2^{\circ}$ stade qui subira un développement important chez le vertébré ; d'autre part, la migration transitoire dans la muqueuse est une migration très précoce, intéressant surtout le $2^{\circ}$ stade.

Chez Ascaridia galli (Schrank), étudié par Tugwell et Ackert (1952), cette migration est devenue inutile ; pendant les $2^{\circ}$ et $3^{\circ}$ stades, la croissance est la même pour les larves qui ont migré et pour celles qui sont restées libres dans le tube digestif; mais ensuite (c'est-à-dire pour le $4^{\circ}$ stade), cette migration devient nuisible ; on a montré que les larves grandissent plus vite dans la lumière de l'intestin que dans la muqueuse intestinale.

Ce cycle est donc, malgré son apparente simplicité, le résultat d'une longue évolution.

\section{Il y a un hôte intermédiaire, à séclusion forte, infesté par le premier stade larvaire.}

\section{Gas de Subulura.}

Ici, l'infestation de l'hôte intermédiaire a lieu avec le premier stade larvaire.

L'hôte intermédiaire est cette fois un invertébré à séclusion forte (Arthropodes), à l'intérieur duquel les trois premiers stades larvaires évoluent.

Le cycle n'a aucune tendance à se simplifier; il s'établit au contraire des migrations tardives qui conduiront les adultes dans les différents tissus de l'hôte définitif. 


\section{Epidémiologie des Ascaridiens de nos pays}

Nous nous proposons maintenant de passer en revue les divers Ascaridiens susceptibles d'entrer en contact avec l'homme dans nos pays.

Pour des raisons d'ordre biologique, nous avons vu que ce sont principalement les espèces migrant à l'état larvaire chez des petits vertébrés à sang chaud comme hôtes intermédiaires qui pouvaient infester aecidentellement notre organisme.

Ces mêmes espèces ont toutes pour hôte définitif un vertébré de régime carnivore qui s'infestera en dévorant l'hôte intermédiaire. C'est done chez ces vertébrés de régime carnivore que nous trouverons les éventuels agents de dissémination du parasite.

On peut considérer successivement le cas des Serpents, celui des Oiseaux, celui des Mammifères.

\section{Cas des Serpents éventuellement vecteurs}

Plusieurs Serpents de nos régions pourraient peut-être jouer un rôle important dans la dissémination des parasites.

En tout premier lieu, la Vipère de France, représentée par deux espèces, Vipera aspis (Linné) et Vipera berus (Linné), si communes dans de nombreuses régions, présente les conditions voulues; en effet, ce Serpent fréquente les jardins, les champs cultivés, les potagers et dépose ses excréments sur le sol. Il peut donc souiller les légumes.

Son régime, d'autre part, est exclusivement carnivore; elle se nourrit principalement de petits Rongeurs, Souris, Mulots, Campagnols, qui sont aussi très communs partout.

Bien que ne connaissant pas encore le cycle des Hexametra, Ascaridiens parasites des Vipères, il semble que le genre Hexametra, normalement parasite de Reptiles, ne puisse se développer directement (A.-G. Chabaud, 1955).

On peut admettre, comme c'est le cas pour Ophidascaris, autre parasite de Serpent, que l'Hexametra ait besoin d'évoluer chez le petit Rongeur, où les larves effectueraient des migrations jusqu'au $3^{\circ}$ stade qui serait alors ingéré avec la Souris par la Vipère.

Dans ce cas, l'homme pourrait se trouver intercalé dans le cycle à la place du petit Rongeur ; des œufs d'Hexametra déposés sur un 
légume, salade par exemple, mal lavée, sont ingérés par l'homme ; les petites larves du $2^{\circ}$ stade migrent et muent jusqu'au $3^{e}$ stade, occasionnant des troubles variés au cours des migrations tissulaires.

Certaines Couleuvres présentent une écologie comparable.

La Couleuvre verte et jaune ou Zamenis, Coluber jugularis (Linné), des régions méridionales, est fréquemment rencontrée dans les régions cultivées. Extrêmement vorace et plus rapide que la Vipère, son régime se compose aussi de petits Mammifères rongeurs qu'elle capture adroitement dans leurs terriers.

On trouve chez ce Serpent Hexametra waterstoni.

La Couleuvre de Montpellier, Malpolon monspessulana (Hermann), autre grande Couleuvre, présente le même régime et les mêmes mœurs. Elle est parasitée par Hexametra sewelli.

On pourrait encore citer, bien que plus rares, la Couleuvre d'Esculape, Elaphe longissima Laurenti), et la Couleuvre à échelons, Elaphe scalaris (Schinz).

Nous éliminons volontairement les a'tres Colubridés de nos pays, gui ont des mœurs aquatiques et un régime composé de Poissons ou de Batraciens, comme dans le cas de la Couleuvre à collier (Natrix natrix Linné) et de la Couleuvre vipérine, Natrix viperına (Latreille); de même, la Couleuvre coronelle, Coronella austriaca (Laurenti), de taille trop faible, ne peut cntrer dans la catégorie qui nous intéresse ici.

\section{Cas des Oiseaux éventuellement vecteurs}

Le ròle de vecteurs d'Ascaridiens hétéroxènes des Rapaces de nos régions parait facile à comprendre.

Ces Oiseaux ont presque toujours un terrain de chasse avoisinant ıes cultures : c'est le cas chez le Faucon crécerelle Falco tinnunculus (Linné), l'Epervier Accipiter nisus (Linné), la Bııe variable Buteo buteo (Linné), les Busards Circus, pour ne citer que les plus communs des Rapaces diurnes, et, parmi les Rapaces nocturnes, la Chouette chevêche Athene noctua (Scopoli) et la Chouette effraie Tyto alba alba (Scopoli).

Tous ces Oiseaux font une grande consommation de petits Mammifères, notamment d'Insectivores qu'ils capturent dans les chainps.

On trouve chez ces Rapaces plusieurs espèces de Porrocæcum, dont le cycle évolutif exige le passage des stades larvaires dans un Mammifère insectivore, Musaraigne ou Taupe. 
Il est probable que ces hòtes intermédiaires eux-mèmes s'infestent en dévorant un invertébré comme le Ver de terre, par exemple, premier hôte intermédiaire possible.

L'homme pourrait se trouver intercalé dans le cycle, à la place de l'Insectivore, par ingestion accidentelle d'un Ver de terre, ce qui est possible, avec une feuille de salade, mais d'éventualité peu probable.

\section{Cas des Mammifères éventuellement vecteurs}

L'infestation par l'intermédiaire de Mammifères Carnivores sauvages est certainement la plus fréquente.

En effet, ces animaux sont communs partout et peuplent pendant la nuit les champs et les jardins, détruisant un grand nombre de petits Rongeurs.

Parmi les Mustelidés, l'Hermine Mustela herminea L. et la Belette Mustela nivalis L. sont les plus répandues.

Ces petits fauves chassent souvent très près des habitations el leurs excréments peuvent facilement souiller les légumes. D'autres espèces, plus rares, peuvent également être citées : la Fouine Martes foina (Erxleb.), le Putois Mustela putorius L., le Blaireau Meles meles $\mathrm{L}$.

Ces Mustelidés sont fréquemment porteurs de l'Ascaris columnaris, qui pourra facilement infester l'homme à la place du petit Rongeur. Le Blaireau est souvent parasité par Toxocara melis.

Le cas de l'Ascaris du Chien, Toxocara canis, et celui du Chat, Toxocara mystax, sont certainement les plus étudiés et représentent la majorité des cas observés en clinique; ils peuvent être transmis à l'homme, non seulement par le Chien et le Chat domestique, mais aussi par les Renards qui abondent dans certaines régions où leur terrain de chasse borde les cultures. De mème pour Toxascaris leonina, qui se retrouve chez le Renard, le Chien domestique, le Chat, etc...

Nous n'envisagerons pas ici le cas de l'Ascaris du Cheval, Parascaris equorum (Goeze), de celui du Veau, Neoascaris vitulorum (Goeze), ni celui du Porc, Ascaris suum, qui, bien que très répandus chez nos animaux domestiques, ne semblent pas être pathogènes pour l'homme, comme nous l'avons vu plus haut. 


\section{Syndrome clinique : historique, discussion}

A la suite de nombreuses observations publiées dans plusieurs pays, sur un syndrome allergique d'origine imprécise, le terme de Larva migrans viscérale fut proposé en 1952 par Beaver et ses collaborateurs.

Ce syndrome était caractérisé par « une éosinophilie chronique * extrême, des lésions de granulomes éosinophiles avec hépatomé* galie, un certain degré d'infiltration pulmonaire ; il atteignait de * préférence les jeunes enfants et était causé par des larves d'As« carides du Chien et du Chat du genre Toxocara, peut-être par « d'autres ».

Déjà de nombreux cas d'Eosinophilie importante survenus chez des enfants, associés à des symptômes variés : fièvre, infiltrats pulmonaires, hépatomégalie, étaient signalés par Fulleborn en 1921 et Chandler en 1925 , et bien souvent on n'en décelait pas les causes.

Ces cas étaient attribués, soit à la Maladie de Weingarten, au Syndrome de Frimodt-Moeller, à l'Eosinophilie familiale, etc., mais les premiers pas vers la découverte de larves de Toxocara furent faits par Perlingiero J. G. et Gyorgy P., qui, en 1947, avaient rapporté un cas d'Eosinophilie chronique, avec nécrose du foie, des infiltrations pulmonaires, de l'anémie associée avec une infestation ascaridienne chez un enfant de 2 ans. La durée des troubles s'étendait sur quinze mois, les globules blancs atteignirent le chiffre de 55.000, avec $45 \%$ d'éosinophiles.

Zuelzer W. et Apt L., en 1949, rapportèrent plusieurs cas, où des lésions viscérales disséminées étaient associées à une éosinophilie extrême :

Cas de Zuelzer et Apt

\begin{tabular}{|c|c|c|c|}
\hline & Enfant & Globules blancs & Eosinophiles \\
\hline 25 mois & $\ldots \ldots \ldots \ldots$ & 24.800 & $40 \%$ \\
\hline 18 mois & $\ldots \ldots \ldots \ldots$ & 43.000 & $36 \%$ \\
\hline 38 mois & $\ldots \ldots \ldots \ldots$ & 11.900 & $20 \%$ \\
\hline 24 mois & $\ldots \ldots \ldots \ldots \ldots$ & 98.000 & $87 \%$ \\
\hline 24 mois & $\ldots \ldots \ldots \ldots \ldots$ & 50.100 & $52 \%$ \\
\hline 36 mois & $\ldots \ldots \ldots \ldots \ldots$ & 35.800 & $40 \%$ \\
\hline 36 mois & $\ldots \ldots \ldots \ldots \ldots$ & 8.800 & $21 \%$ \\
\hline
\end{tabular}


En 1950, Beaver P. C. et coll. découvrirent des larves par biopsie du foie. La même année, Mercer et coll. citaient le cas d'un enfant de 2 ans ayant des manifestations viscérales et une éosinophilie de $62 \%$.

En 1951, un enfant de 15 mois, cité par Behrer M. R. et coll., présentait une éosinophilie de $50 \%$. Chez ces deux derniers cas, une biopsie hépatique était positive et montrait la présence de larves.

En 1952, Beaver P. C. et coll. publient encore quatre cas analogues, et rapportent le cas décrit par Atmar en 1940, où le malade était un adulte de 25 ans, qui montra à l'opération la présence de granulomes éosinophiliques du foie.

\section{Cas de Beaver}

\begin{tabular}{ccc}
\hline \hline \multicolumn{1}{c}{ Enfant } & Globules blancs & Eosinophiles \\
\hline 16 mois $\ldots \ldots \ldots \ldots \ldots$ & 45.000 & $70 \%$ \\
26 mois $\ldots \ldots \ldots \ldots \ldots$ & 21.700 & $62 \%$ \\
30 mois $\ldots \ldots \ldots \ldots \ldots$ & 50.000 & $30 \%$ \\
19 mois $\ldots \ldots \ldots \ldots \ldots$ & 20.000 & $30 \%$ \\
\hline \hline
\end{tabular}

En 1953, dans le cas de Dent et Carréra, le malade avait 49.200 globules blancs, avec $75 \%$ d'éosinophiles.

Dans les cas de Fellers (1953); chez deux enfants de 18 mois et 6 ans, on trouvait 15 à 18.000 globules blancs et de 30 à $80 \%$ d'éosinophiles. Dans les deux cas, l'existence de Toxocara fut confirmée.

Brill, Churg et Beaver rapportent un cas se rapprochant de celui de Perlingiero et Gyorgy, Zuelzer et Apt, Mercer et coll., et Beaver, mais ici l'évolution en fut encore plus grave.

Il s'agissait d'un enfant de 2 ans, sans aucun symptôme pulmonaire, avec état subcomateux et fièvre. Il présentait une formule sanguine très troublée : 31.500 leucocytes et $40 \%$ d'éosinophiles. L'enfant mourut après une courte maladie, de plus en plus léthargique, et se plaignant de douleurs abdominales peu avant l'issue fatale.

A l'autopsie, on trouva les poumons congestionnés, avec, à leur surface, de nombreux nodules. Le cœur, augmenté de volume, montrait des nodules semblables, ainsi que le foie.

Une seule larve fut trouvée dans le poumon, appartenant au genre Toxocara, mais on ne put distinguer s'il s'agissait de $T$. canis ou $T$. cati.

L'étude des lésions montra des épisodes d'invasions successives, ce qui expliquerait, d'après ces auteurs, l'hypersensibilisation, produite par formation d'antigènes provoquant un choc disproportionné au nombre de parasites.

Wilburn et Ernst, de même, en 1953, citent un cas rapporté par Zuelzer, dont la terminaison fut fatale. Il s'agissait d'un enfant de 3 ans, qui mourut quelques heures après son admission à l'hôpital, après des troubles respiratoires, des convulsions, une fièvre élevée. 
L'autopsie montra l'hyperplasie éosinophile de la moelle osseuse, et le granulome éosinophile typique dans le foie.

Ces auteurs considèrent que ces migrations larvaires dans le foie et les autres viscères représentent probablement le stimulus antigénique qui produit la réponse éosinophilique dans tous les cas.

En 1956, Henier D. C. et Kevy S. V. étudièrent trois cas simultanés dans une même famille chez trois sœurs : l'un d'entre eux fut presque fatal, un autre eut une-forme très bénigne, le troisième, asymptomatique, seulement décelé par le chiffre des éosinophiles. Dans les trois cas le chiffre des gamma-globulines était élevé.

Dans les deux cas de Karpinski F. E. et Everts Suarez E.A., ef Sawitz W. G., il s'agissait aussi d'enfants en-dessous de 3 ans, ayant ingéré des œufs de Toxocara canis avec des aliments souillés.

Si les lésions pulmonaires et hépatiques sont les plus fréquemment rencontrées, il existe cependant dans la littérature médicale des cas de localisation très différente.

Ainsi, en 1950, Wilder avait trouvé des larves dans des yeux énucléés pour rétinoblastome présumé dans 24 cas.

Beautyman (cité par Blanch, 1956), en 1951, lors d'une autopsie d'un malade mort de poliomyélite, découvrit un grand nombre de larves dans le cerveau.

Un cas, rapporté en 1956 par Winckel W. E. F. et Treurniet A. E., montre une infestation humaine par une autre espèce d'Ascaridien : Lagochilascaris minor Leiper.

Il s'agissait d'une infestation par un Nématode parasite intestinal de Félidés sauvages de l'Amérique du Sud.

En 1952, une femme noire présenta une infiltration de la peau du côté droit du cou, près de la limite latérale de la glande thyroïde.

Pensant à la possibilité d'un cancer de la thyroïde, une biopsie fut faite, dans laquelle furent trouvées des sections d'un Nématode; les tissus avoisinants montraient une réaction inflammatoire.

Lorsque, quelques jours plus tard, on changea le pansement, un Ver mort, de $1,5 \mathrm{~cm}$. de long environ, fut trouvé, mais l'identification en fut impossible. La blessure guérit rapidement, et la malade retourna chez elle.

Six mois après, elle consultait à nouveau, avec un abcès du côté droit du cou à l'endroit de la première intervention. Quand l'abcès fut ouvert quelques petits Vers furent recueillis. Tout l'abcès fut extirpé, la section transversale montra une cavité de la taille d'une noisette, remplie de plusieurs centaines de Nématodes, 900 environ.

La blessure guérit sans complications. Les examens de laboratoires montrèrent 6.400 globules blancs, avec $11 \%$ d'éosinophiles; cette ma- 
lade ne portait pas de parasitose décelée par examen des selles, n'ètait pas atteinte de Paludisme, et la recherche de Microfilaires sanguines resta négative.

Cette malade revint consulter au début de 1954, avec un très gros abcès, toujours situé au même endroit, et s'étendant latéralement et dorsalement. Des radiographies montrèrent que quelques cellules mastoïdiennes du côté atteint étaient touchées.

Il est probable que la malade a été infestée par ingestion des æufs du parasite, transmis par les excréments d'un Fèlidé souillant ses aliments. Les auteurs de cette observation signalent que Leiper, en 1909, avait déjà trouvé le parasite dans des abcès sous-cutanés chez des habitants de Trinidad.

Pawan, en 1926, Bonne et Ortlepp, Verhagen en 1921, rapportèrent de mème plusieurs cas ; dans le cas observé par Verhagen, Lagochilascaris minor fut extrait d'un abcès mastoïdien.

Nassy (1954) trouva aussi ce ver dans un abcès mastoüdien et dans un abcès de l'amygdale, à Trinidad.

Il est très possible que cette parasitose ne soit pas rare dans ces pays, mais il est très difficile d'avoir une idée précise sur sa répartition, en raison des contacts difficiles avec les indigènes.

Ce cas est particulièrement intéressant, car il met en évidence deux notions jusqu'ici peu reconnues : d'une part, l'infestation par un Ascaridien autre que Toxocara canis ou Toxocara cati; d'autre part, l'infestation d'un sujet adulte et non plus seulement de très jeunes enfants comme jusqu'ici.

L'étude de la Biologie des Ascaridiens, que nous avons rappelée, avait déjà montré la possibilité d'une infestation de l'homme par d'autres Nématodes parasites d'animaux sauvages, et il nous paraît utile d'attirer l'attention des cliniciens sur la possibilité de cas analogues. En effet, on admet facilement que, par l'intermédiaire des légumes des cultures maraìchères, puissent se produire des infestations accidentelles.

Il parait très probable que nombre de cas cliniques qui ne font pas leur preuve soient en fait dus à de tels accidents, et, bien souvent, les cliniciens, mal informés de ces faits, concluent prématurément à l'absence de Parasitose quand ils n'ont pas découvert d'œufs à l'examen coprologique ; nous prendrons pour exemple un extrait d'une thèse de médecine récente (S. Petit Jaumet, 1957), où l'auteur, discutant un diagnostic difficile d'asthme compliqué, concluait: " Nous n'étions pas devant un syndrome de Loeffler * typique puisque les infiltrats pulmonaires ont persisté pendant * des mois et les signes cliniques étaient sévères. 
«L'étiologie parasitaire a pu être éliminée par des examens de " selles répétés dans les délais voulus. 》

D'autre part, le syndrome de Larva migrans a été décrit comme un syndrome de l'enfant de 6 mois à 4 ans; le cas décrit par Atmar en 1940 et celui de Winckel et Treurniet montrent que l'adulte, bien que plus rarement atteint, n'en est pas exempt.

Quand il ne s'agit que des Ascaris du Chien et du Chat, l'enfant est certainement plus souvent infesté, étant souvent en contact plus étroit avec ces animaux familiers.

Les très jeunes enfants, suçant tout ce qu'ils trouvent, on comprend facilement les possibilités d'infestations réalisées sur le sol des jardins, les plages publiques, les squares...

Mais, quand il s'agit d'autres hòtes vecteurs que les Chiens ou les Chats, l'infestation pourra se produire au hasard, à n'importe quel âge, par contamination alimentaire de légumes souillés par exemple.

\section{Signes cliniques}

Nous avons vu que cette maladie pouvait atteindre les adultes comme les enfants, bien qu'elle soit plus souvent diagnostiquée chez les enfants.

Les signes cliniques sont très polymorphes, la maladie pouvant tantôt se limiter à un seul symptôme, tantòt à une association. Chez certains, c'est une éosinophilie constatée lors d'un examen systématique qui oriente les recherches; notamment, une éosinophilie en plateau persistant six mois peut ètre le seul signe en faveur d'une Ascaridiose. Ailleurs, c'est une fièvre intermittente, une perte d'appétit et de poids, de la toux accompagnée de douleurs musculaires, articulaires ou abdominales; dans certains cas, on trouve des éruptions cutanées, de l'œdème de Quincke.

Les signes pulmonaires sont plus fréquents chez l'adulte, ils se traduisent par des quintes de toux ou des crises asthmatiformes. Radiologiquement, on peut retrouver des ombres assez étendues à contours irréguliers, soit des foyers de condensation arrondis, soit des ombres à topographie lobaire ; mais, quelle que soit la forme de ces infiltrats, leur caractéristique est d'être fugaces, et ils disparaissent le plus souvent en une dizaine de jours.

L'hépatomégalie, symptôme le plus fréquent chez les jeunes enfants, est dans tous les cas le signe le plus constant. 
Elle peut manquer dans les cas bénins, mais très souvent le foie peut dépasser le rebord costal de trois travers de doigt.

Elle rétrocède parfois rapidement; ainsi, dans le cas relaté par Morin (1956), le foie reprit ses dimensions normales deux mois après le début des troubles.

Les lésions cérébrales et oculaires, bien que plus rares, peuvent se rencontrer. A ce propos, il est intéressant de constater que, suivant l'espèce, les larves d'Ascaridiens se localisent de préférence dans un organe plutôt qu'un autre.

Ainsi, J.F. A. Sprent, en 1955, a montré expérimentalement, en faisant ingérer des œufs embryonnés de Toxocara canis, T. mystax, Toxascaris transfuga et Ascaris columnaris à des Souris de laboratoire, que les larves de $T$. canis se trouvaient en plus grand nombre dans le cerveau de la Souris que les autres espèces, mais qu'elles n'y occasionnaient que peu de dommages, leur taille étant très petite.

Au contraire, les larves d'Ascaris columnaris du Furet occasionnaient des troubles sérieux à cause de leur grande taille dans leur passage à travers l'encéphale.

J.D. Tiner, en 1952, était arrivé à des conclusions analogues : en constatant des troubles cérébraux fréquents chez les Rongeurs sauvages infestés expérimentalement.

M. H. D. Smith et P. C. Beaver, en 1953, infestèrent expérimentalement deux enfants déficients mentaux avec 200 œufs de T. canis; ils restèrent cliniquement asymptomatiques, mais développèrent une éosinophilie pendant plus de 13 mois.

Par contre, des Souris infestées par la même dose d'œufs furent étudiées un an après ; aux premiers stades, les larves furent trouvées surtout dans le foie et les poumons, mais, aux stades plus tardifs, principalement dans le cerveau.

Pour ces auteurs, les nombreuses manifestations neurologiques associées à l'Ascaridiose pourraient bien être dues à la présence de larves dans le S.N.C., plutôt qu'à un effet toxique ou allergique de l'Ascaris lumbricoides.

\section{Anatomo-pathologie}

Dans son travail d'ensemble sur la Larva migrans viscérale, le Professeur Henri Galliard (1957) résume ainsi le type histopathologique de la lésion : 
« Le type histopathologique de la lésion viscérale est le granulome éosinophilique allergique (Churg et Strauss). Dans les lésions récentes, c'est-à-dire le tunnel creusé par la larve dans le parenchyme, le centre nécrosé est constitué par des cellules hépatiques nécrosées et des éosinophiles désintégrés, et du tissu conjonctif fibrinö̈de, le tissu voisin est infiltré massivement par des éosinophiles et contient des cristaux de Charcot-Leyden.

«Dans les lésions plus anciennes, et dans celles qui contiennent une larve dégénérée, des cellules géantes, du type " corps étranger", sont visibles dans la zone des cellules épithélioïdes, et le granulome entier peut être entouré d'une capsule fibreuse.

« Les granulomes sont dispersés dans le tissu conjonctif des différents organes, souvent centrés par un vaisseau, le plus souvent une veinule. Aussi bien les artères que les veines peuvent être intéressées par le processus.

«La nécrose veineuse est fréquente dans les poumons et le rein; le processus débute par une phlébite et la veine lésée est le plus souvent au centre du granulome. »

\section{Pronostic et traitement}

Les troubles provoqués par les migrations tissulaires sont le plus souvent sans réelle gravité ; on constate fréquemment la rétrocession de tous les symptômes et la formule sanguine redevient normale en un temps souvent assez long ; ainsi, dans le cas de Zuelzer et Apt (1949), la guérison ne survint que trois ans après le début de la maladie; il semble, en effet, que les larves enkystées finissent par mourir dans les tissus humains, où elles ne peuvent parvenir à maturité.

Cependant, suivant leur localisation dans l'organisme, ces lésions pourront aussi avoir des suites fâcheuses ou même fatales, dans le cas où les larves se trouvent dans un œil ou dans l'encéphale.

Le traitement est en général décevant: les dérivés de la Pipérazine (Hétrazan) essayés se sont montrés inefficaces ; par contre, il semblerait que l'association Cortisone + Antibiotiques soit indiquée, mais leur action n'est pas confirmée.

\section{Conclusions}

Après avoir étudié la Biologie des Ascaridiens, nous savons que les Ascaridiens hétéroxènes ont besoin le plus souvent d'hôtes intermédiaires dans lesquels évoluent leurs larves; nous savons que ces 
hôtes intermédiaires sont le plus souvent peu spécifiques, contrairement à ce qui se produit pour l'hôte définitif; nous avons vu aussi que le passage des larves dans les tissus de l'hôte intermédiaire, lorsqu'il correspond à une phase de métabolisme actif, ne se faisait jamais sans signes cliniques.

Il nous est facile d'établir un rapprochement entre ces faits et la réalité des cas cliniques observés. Dans la plupart des cas relatés, il s'agissait d'Ascaridiens relativement communs du Chien et du Chat, dont les jeunes larves évoluaient dans les tissus humains, ceux-ci jouant le rôle d'hôte intermédiaire.

Cependant, nous avons vu le cas très intéressant d'infestation par le Lagochilascaris minor, où ce parasite des Félidés sauvages fut retrouvé à plusieurs reprises chez des êtres humains.

Nous pensons qu'il est possible que des faits analogues se reproduisent souvent, l'homme se contaminant par ingestion accidentelle d'œufs d'Ascaridiens d'animaux sauvages; nous avons donc recensé les espèces de Vertébrés les plus communs dans nos pays, Serpents, Oiseaux et Mammifères, qui, par leur fréquence et leur vie à proximité des cultures, pouvaient par leurs excréments nous transmettre leurs parasites intestinaux.

\section{BibliogRAPHIE}

Axos., 1949. - Pig as Vector of Ascaris. Brit. Medic. Journ. (Questions el Answers), 1, (4607), 735.

Atmar (R. C.), 1940. - Familial eosinophilia : Report of case with biopsy of liver. J.A.M.A., 115-449.

Beaver (P. C.), 1952, Snyder (C. H.), Carrera (G. M.), Dent (J. H.) et Lafferty (J. W.). - Chronic cosinophilia due to Visceral larva migrans, Report of three cases (Tox, canis). Pediatrics Springfield, (1), 9, (1), 7-19.

BeAver (P. C.), 1954. - Parasitic diseases of animals and their relation to public health. Veterinary Medicine, 49, (5), 199-202, 205.

Beaver (P. C.), 1956. - Larva migrans. Experimental Parasitology, N.-Y., nov., $\mathrm{V}, 5, \mathrm{n}^{\circ} 6,587-621,1$ fig.

Behren (M. R.), 1951. - Hypereosinophilia with eosinophilic granuloma of the liver associated with ascaris infestation. Pediatrics, $38, \mathrm{n}^{\circ} 5$, mai 1951 , p. $635-640$.

Blaxch (M.), 1956. - Eosinophilia with hepatomegaly. Medical Journal of Australia, 43e Year, 2, (5), 184-185.

Brill (R.), Chung (J.) et Beaver (P. C.), 1953. - Allergic granulomatosis associated with Visceral larva migrans. Case report with autopsy findings dog Toxocara infection in a child. American Journal of Clinical Pathology, 23, (12), 1208-1215.

Brumpt (E.). - Précis de Parasitologie, Paris. 
Снаваdd (A. G.), 1955. - Essai d'interprétation phylétique des cycles évolutifs chez les Nématodes parasites de vertébrés, conclusions taxonomiques. Ann. de Parasit. hum. et comparée, $\mathrm{XXX}, \mathrm{n}^{\circ}$ 1-2.

Chander (A. C.), 1925a. - The helminthic Parasites of cats in Calcutta and relations of cats to human helminthic infections. Indian J. Med. Research, 13, 213-228.

Ehrenford (F. A.), 1957. - Canine Ascariasis as a potentiai source of visceral larna migrans. American Journal of Trop. Medicine and Hygiene, 6, (1), 166,170 .

Fellers (F. X.), 1953. - Agglutination studies in visceral larva migrans. American J. of Diseases of Children, 86, $\mathrm{n}^{\circ} 5,767-771$.

Fullerors (F.), 1921. - Askarisinfection durch Verzehren eingekapselter Larven und über gelungene intrauterine Askarisinfection. Arch. Schiffs-u-Tropen Hyg., 25, 367-375.

Galı.ıиn (H.), 1957. - Larva migrans viscérale. Presse médicale, $\mathrm{n}^{\circ} 39$, 15 mai $1957,916-918$.

Heiner (D. C.) et Kevy (S. V.), 1956. - Visceral larva migrans. Report of the syndrome in three siblings, 254, (14), 629-636.

Karpinsky (F. E. Jr), Everts-Suarez (E. A.) et Sawitz (W. G.), 1956. - Larval granulomatosis (Visceral larva migrans). July, V, 92, $\mathrm{n}^{\circ} 1,34-40$.

Mendheim (H.), Scheiv (G.) et Schmidt (J.), 1952. - Die seltenren SpulwurmInfektionen beim Menschen. Zeitsch. für Trop. Mediz, und Parasit., Stuttgart, Febr. 1952, 3, (3), 368-371.

Mercer (R. D.), Luxd (H. Z.), Blomfield (R. A.) et Caldwell (F. E.), 1950. Larval Ascariasis as a cause of chronic eosinophilia with visceral manifestation. American Journal of Diseases of Children Chicago, 80, (1), 46-58.

Morin (1956). - Contribution à l'étude de l'Ascaridiose larvaire. Thèse Paris, 1956, dactylographiée.

Mosgovor (A. A.), 1953. - Principles of Nematodology, edited by K. I. Skrjabin, vol. II. Moscow Izdatelstvo Akademii, Nauk S.S.S.R., 351 pages.

Nichol.s (R. L.), 1956. - The Etiology of visceral larva migrans. I. Diagnostic, Morphology of Infective second stage Toxocara larva. Journ. of Paras.. $\mathrm{V}, 42 . \mathrm{n}^{\circ} 4,349-362$.

Osche (G.), 1955. - Ueber Entwicklung, Zwischenwirt und Bau von Porrocæcum talpe, Porrocacum ensicaudatum und Habronema mansioni (Nematoda). z. f. Parasitenkunde, V, 17, S. 144-164.

Perlingierio (J.) and Gyorgy (P.), 1947. - Chronic eosinophilia : Report of a case with necrosis of the liver, Pulmonary infiltrations, anemia and Ascaris infestation. Am. J. Diseases children, 73, 34-43.

Petit-Jaumet (S.), 1957. - Contribution à l'étude des pneumopathies avec éosinophilie sanguine. Thèse Paris, $\mathrm{n}^{\circ} 808$.

Sмith (M. H. D.) et Beaver (P. C.), 1953. - Persistence and distribution of Toxocara larve in the tissues of children and mice. Pediatrics, Springfield, 12, (5), 491-497.

SPRENT (J. F. A.), 1950. - Observations on the life history of Ascaris columnaris. Journal of Parasitology, 36, (6, sect. 2), Suppl. 1, p. 29.

Sprent (J. F. A.), 1951. - Observations on the migratory activity of the larva of Toxascaris transfuga (Rud. 1819). (Baylis et Daubney, 1922). Journal of Parasitology, 37, (3), 326-327.

Sprent (I. F. A.), 1952. - On the migratory behaviour of the larvæ of various 
Ascaris species in white mice. Distrib. of larva in tissues. Journal of infectious diseases, 90, (2), 165-176.

SPRENT (J. F. A.), 1953. - Intermediate hosts in Ascaris infections. Journal of Parasitology, 39, (4, sect. 2), suppl. p. 38.

SPRENT (J. F. A.), 1955. - On the invasion of the central nervous system in Ascariasis. Parasitology, 45, $\mathrm{n}^{\circ}$ 1-2, 41-55.

Spnent (J. F. A.), 1956. - The life history and development of Toxocara cati (Schrank 1788) in the domestic cat. Parasitology, 46, (1-2), 54-78.

Texeina de Freitas (J. F.) et Lent (H.), 1946. - Infestaçao de apaiaris Astronotus ocellatus (Agassiz) peio Nematodeo Goezia spinulosa (Diesing, 1839). Ke:). Brasil. Biol., VI, 215-222, 1946, fig. 1-7.

Tномаs (L. J.), 1937. - Life cycle of Rhaphidascaris canadensis Smedley 1933, an nematode from the pike, Esox lucius. J. Parasit., XXIII, p. 572.

1937b. - On the life-cycle of Contracecum spiculigerum. J. Parasit., XXIII, p. $429-431$.

TINER (J. D.), 1949. - Preliminary observations on the life history of Ascaris columnaris. J. Parasit., 35 (6, sect. 2), Suppl. p. 13.

Tiner (J. D.), 1952. - Speciation in the genus Ascaris : additional experimental and morphrological data. Journal of Parasitology, 38 (4, sect. 2), Suppl. p. 27 .

Tiner (J. D.), 1953. - Fatalities in rodents caused by larval Ascaris in the central nervous system. Jonrnal of Mammalogy, 34, (2), 153-167.

Tugwel. (R. L.) et Ackert (J. E.), 1952, - On the tissue phase of the life cycle of the fowl nematode Ascaridia galli (Schrank). J. Parasit., XXXVIII, 1952, 277-288, fig. $1-2+2 \mathrm{pl}$.

Wilburn (C. L. Jr) et ErNst (K. F.), 1953. - Eosinophilia-hepatomegaly syndrome of infants and young children. Report of a case due to invasion of liver by nematodes larvæ. Pediatrics Springfield, 11, (4), 358-367.

WrLder (H. C.), 1950. - Nematode endophtalmitis. Tr. Am. Acad. Opht., nov.déc., p. 99.

Wingkel (W. E. F.) et Treurniet (A. E.), 1956. - Infestation with Lagochilascaris minor (Leiper) in Man. Documenta de Medicina geographica et tropica Amsterdam, 8, (1), 23-28.

ZURLzer (W.) and APT (L.), 1949. - Disseminated visceral lesions associated with extreme eosinophilia. Am. J. Diseases children, 78, 153, 181. 